\title{
Parallel Concatenation of Non-Binary Linear Random Fountain Codes with Maximum Distance Separable Codes
}

\author{
Francisco Lázaro Blasco, Student Member, IEEE, Giuliano Garrammone, Student Member, IEEE, Gianluigi Liva, \\ Member, IEEE
}

\begin{abstract}
The performance and the decoding complexity of a novel coding scheme based on the concatenation of maximum distance separable (MDS) codes and linear random fountain codes are investigated. Differently from Raptor codes (which are based on a serial concatenation of a high-rate outer block code and an inner Luby-transform code), the proposed coding scheme can be seen as a parallel concatenation of a MDS code and a linear random fountain code, both operating on the same finite field. Upper and lower bounds on the decoding failure probability under maximum-likelihood (ML) decoding are developed. It is shown how, for example, the concatenation of a $(15,10)$ ReedSolomon (RS) code and a linear random fountain code over a finite field of order $16, \mathbb{F}_{16}$, brings to a decoding failure probability 4 orders of magnitude lower than the one of a linear random fountain code for the same receiver overhead in a channel with a erasure probability of $\epsilon=5 \cdot 10^{-2}$. It is illustrated how the performance of the novel scheme approaches that of an idealized fountain code for higher-order fields and moderate erasure probabilities. An efficient decoding algorithm is developed for the case of a (generalized) $\mathrm{RS}$ code.
\end{abstract}

Index Terms-Fountain codes, maximum distance separable codes, maximum likelihood decoding, erasure correction.

\section{INTRODUCTION}

$\mathbf{E}$ FFICIENT reliable multicasting/broadcasting techniques have been investigated during the past thirty years [1] and especially during the past decade [2]-[10]. Perhaps, the most successful approach to reliable multicast deals with the socalled fountain codes [2]. Consider the case where a sender (or source) needs to deliver a source block (e.g., a file) to a set of $N$ receivers. Consider furthermore the case where receivers are affected by packet losses. In this scenario, the usage of an Automatic Retransmission Query (ARQ) protocol can result in large inefficiencies, since receivers may loose different packets, and hence a large number of retransmissions would crowd the downlink channel. When a fountain code is used, the source block is split in a set of $k$ source packets,

This work was presented in part at IEEE International Conference on Communications 2011, Kyoto. Francisco Lázaro Blasco, Giuliano Garrammone and Gianluigi Liva are with the Institute of Communications and Navigation, German Aerospace Center (DLR), Oberpfaffenhofen, 82234 Wessling, Germany. Email:\{Francisco.LazaroBlasco,Giuliano.Garrammone, Gianluigi.Liva\}@dlr.de.

This work has been accepted for publication in IEEE Transactions on Communications: Digital Object Identifier 10.1109/TCOMM.2013.09.120834

(c) 2013 IEEE. Personal use of this material is permitted. Permission from IEEE must be obtained for all other uses, in any current or future media, including reprinting /republishing this material for advertising or promotional purposes, creating new collective works, for resale or redistribution to servers or lists, or reuse of any copyrighted component of this work in other works which we will denote as source symbols. The sender computes linear combinations (also referred to as fountain coded packets, or output symbols) of the $k$ source packets and broadcasts them through the communication medium. After receiving $k$ fountain coded packets, the receivers can try to recover the source packets. In case of decoding failure, they will try again to decode after receiving additional packets. The efficiency of a fountain code deals with the amount of packets that a receiver needs to collect for recovering the source block. An idealized fountain code would allow the recovery with a failure probability $P_{f}=0$ from any set of $k$ received packets. Actual fountain decoders need in general to receive a larger amount of packets, $m=k+\delta$, for succeeding in the recovery. Commonly, $\delta$ is referred to as (receiver) overhead of the fountain code, and is used to measure its efficiency. The first class of practical fountain codes are Lubytransform (LT) codes [3]. Among them, random LT codes or linear random fountain codes (LRFCs) [4], [5] deserve a particular attention due to their excellent performance and to the relatively simple performance model. Under maximumlikelihood (ML) decoding, the failure probability of a binary LRFC [4], [5] can be accurately modeled as $P_{f} \sim 2^{-\delta}$ for $\delta \geq 2$. It can be proved that $P_{f}$ is actually always upper bounded by $2^{-\delta}$ [4], [5], [11]. In [6], [9] it was shown that this expression is still accurate for fountain codes based on sparse matrices (e.g., Raptor codes [4]) under ML decoding. In [6], the performance achievable by performing linear combinations of packets on finite fields of order larger than $2\left(\mathbb{F}_{q}, q>2\right)$ was analyzed. For a LRFC over $\mathbb{F}_{q}$, the failure probability under ML decoding is bounded as [6]

$$
q^{-\delta-1} \leq P_{f}(\delta, q)<\frac{1}{q-1} q^{-\delta}
$$

where both bounds are tight already for $q=2$, and become tighter for increasing $q$. The improvement in efficiency obtained by fountain codes operating on fields of order larger than 2 has been analyzed in [6], [10] and has led to recent standardization activities [12]. In [6], [10] it was also shown that non-binary Raptor and LT codes can in fact tightly approach the bounds (1) down to moderate error rates under ML decoding. Thus, (1) can be successfully used to model the performance of common classes of fountain codes. The result is remarkable considering that for Raptor codes, under belief propagation (BP) decoding, both the encoding and decoding 
costs $^{1}$ are $\mathcal{O}(\log (1 / \varepsilon))$ [4, Theorem 5], being $\varepsilon=\delta / k$ the overhead (normalized to $k$ ) needed to recover the source symbols with a high probability. For a LRFC the encoding cost is $\mathcal{O}(k)$ and the decoding cost is $\mathcal{O}\left(k^{2}\right)$, and thus it does not scale favorably with the source block size. However, BP decoding is scarcely used in practical Raptor decoder implementations [13] due its poor performance with source block lengths of practical interest ( $k$ up to few thousands symbols). Efficient ML decoding algorithms based on Gaussian elimination (GE) are usually adopted [13]-[18], for which the decoding cost is $\mathcal{O}\left(k^{2}\right)$, though the fraction of symbols that are recovered with quadratic cost can be kept remarkably small. Similarly, in the short source block length regime, the application of LRFCs under GE decoding is usually considered practical [6], [10].

In this paper, we introduce and analyze a further improvement of the approach proposed in [6], [10] to design fountain codes with good performance for short block lengths. More specifically, a $(n, k)$ maximum distance separable (MDS) code is introduced in parallel concatenation with the LRFC. By doing that, the first $n$ output symbols are the codeword symbols of the MDS code. ${ }^{2}$ We will assume that the MDS linear block code is constructed on the same field $\mathbb{F}_{q}$ as the fountain code. A related rate-less construction was proposed in [19], where a mother non-binary low-density parity-check code was modified by replicating the codeword symbols (prior multiplication by a non-zero field element) and thus by (arbitrarily) lowering the code rate. In our work, the mother code is a MDS code, while additional redundant symbols are produced by a linear random fountain encoder. For the proposed scheme, we illustrate how the performance of LRFCs in terms of probability of decoding failure can be remarkably improved thanks to the concatenation, especially for low to moderate packet loss probabilities. Tight bounds on the decoding failure probability vs. overhead are derived under the assumption of ML decoding. The accuracy of the bounds is confirmed through simulations. An efficient ML decoding algorithm is presented for the case where a (generalized) Reed-Solomon (RS) is used in the concatenation. An analysis for the general case where the MDS code is replaced by any arbitrary linear block code, in a finite rate regime, is provided in the Appendix.

The paper is organized as follows. In Section II the proposed concatenated scheme is introduced. Section III provides an efficient ML decoding algorithm. In Section IV the performance is analyzed and tight bounds on the decoding failure probability are derived, while numerical results are presented in Section V. Conclusions follow in Section VI.

\section{Concatenation of Block Codes With Linear RANDOM FOUNTAIN CODES}

We define the source block $\mathbf{u}=\left(u_{1}, u_{2}, \ldots, u_{k}\right)$ as a vector of source symbols belonging to a finite field of order $q$, i.e., $\mathbf{u} \in \mathbb{F}_{q}^{k}$. In the proposed approach, the source block is first encoded via a $(n, k)$ linear block code $\mathcal{C}^{\prime}$ over $\mathbb{F}_{q}$ with

\footnotetext{
${ }^{1}$ The cost is defined as the number of arithmetic field operations divided by the number of source symbols, $k$.

${ }^{2}$ This represents a crucial difference with Raptor codes, for which the output of the precode is further encoded by a LT Code. Hence the first $n$ output symbols of a Raptor encoder do not coincide with the output of the precode.
}

generator matrix $\mathbf{G}^{\prime}$. The encoded block is hence given by $\mathbf{c}^{\prime}=\mathbf{u} \mathbf{G}^{\prime}=\left(c_{1}^{\prime}, c_{2}^{\prime}, \ldots, c_{n}^{\prime}\right)$. Additional redundancy symbols can be obtained by computing linear random combinations of the $k$ source symbols as

$$
c_{i}=c_{i-n}^{\prime \prime}=\sum_{j=1}^{k} g_{j, i} u_{j}, \quad i=n+1, \ldots, l
$$

where the coefficients $g_{j, i}$ in (2) are picked from $\mathbb{F}_{q}$ with a uniform probability.

The encoded sequence is thus $\mathbf{c}=\left(\mathbf{c}^{\prime} \mid \mathbf{c}^{\prime \prime}\right)$. The generator matrix of the concatenated code has the form

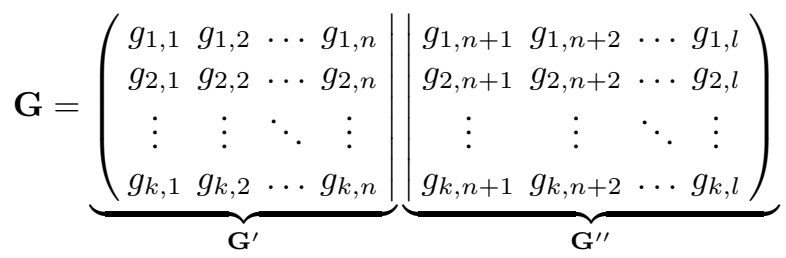

where $\mathbf{G}^{\prime \prime}$ is the generator matrix of the LRFC. Note that, being the LRFC rate-less, the number $l$ of columns of $\mathbf{G}$ can grow indefinitely. The encoder can be seen hence as a parallel concatenation of the linear block code $\mathcal{C}^{\prime}$ and of a LRFC (Fig. 1) and the encoded sequence can be written as $\mathbf{c}=\mathbf{u G}=$ $\left(c_{1}, c_{2}, \ldots, c_{l}\right)$. The proposed construction allows generating infinitely many redundancy symbols. Thus, the encoder may be seen as a modified fountain encoder, whose first $n$ output symbols $\left(c_{1}, c_{2}, \ldots, c_{n}\right)$ correspond to the codeword output by the encoder of $\mathcal{C}^{\prime}$, whereas the following $l-n$ symbols are the output of the LRFC encoder.

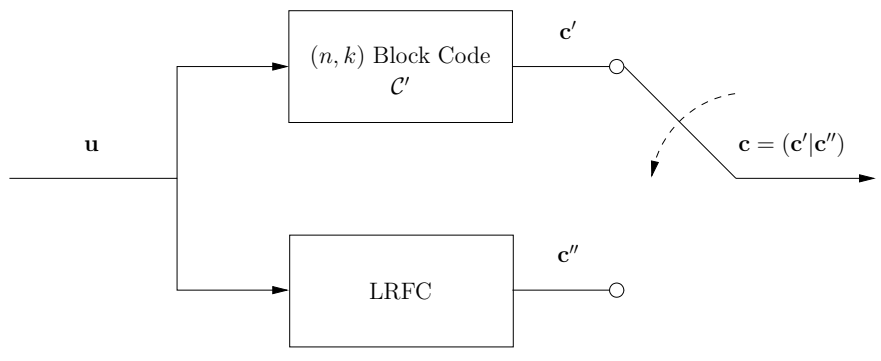

Fig. 1. Fountain coding scheme seen as a parallel concatenation of a $(n, k)$ linear block code and a LRFC.

\section{EFFICIENT DECODING}

We consider a multicast setting, where a number of receivers try to retrieve the source block from the respectively-received output symbols. In this context, the decoder behaves as for a conventional fountain decoder. At each receiver, the correctlyreceived output symbols are forwarded to the decoder. As soon as $k$ output symbols are collected, a decoding attempt is performed. If the decoding is not successful, further output symbols are collected. Whenever an additional output symbol is received, another decoding attempt is performed. In case of successful decoding, the receiver acknowledges the correct reception. The overall number of symbols collected at a receiver is denoted by $m=k+\delta$ (recall that $\delta$ is referred to as the overhead). On the encoder side, as soon as a target success 
rate among the receivers is attained, encoding stops. Note that at each receiver, the $m$ output symbols that are collected may belong to

i) the output of the $\mathcal{C}^{\prime}$ encoder only,

ii) the output of the LRFC encoder only,

iii) both the outputs of the $\mathcal{C}^{\prime}$ encoder and the LRFC encoder. While in the third case there is no different with respect to a classical LRFC case, in the other two cases the structure of the $\mathcal{C}^{\prime}$ generator matrix can be exploited to reduce the decoding complexity, as we will see next. Furthermore, when the channel erasure probability is sufficiently low, the event i) may dominate, leading to a remarkable improvement in the decoding failure probability. In this sense, the proposed scheme provides the same performance of a (universal) LRFC at high channel erasure probabilities, whereas it will enjoy a boost in the efficiency when the channel erasure probability is low. We denote by $\mathscr{J}=\left\{j_{1}, j_{2}, \ldots, j_{m}\right\}$ the set of the indexes on the symbols of $\mathbf{c}$ that have been collected by a specific receiver. The received vector $\mathbf{y}$ is hence given by

$$
\mathbf{y}=\left(y_{1}, y_{2}, \ldots, y_{m}\right)=\left(c_{j_{1}}, c_{j_{2}}, \ldots, c_{j_{m}}\right)
$$

and it can be related to the source block $\mathbf{u}$ as $\mathbf{y}=\mathbf{u} \tilde{\mathbf{G}}$. Here, $\tilde{\mathbf{G}}$ denotes the $k \times m$ matrix made by the columns of $\mathbf{G}$ with indexes in $\mathscr{J}$, i.e.,

$$
\tilde{\mathbf{G}}=\left(\begin{array}{cccc}
g_{1, j_{1}} & g_{1, j_{2}} & \ldots & g_{1, j_{m}} \\
g_{2, j_{1}} & g_{2, j_{2}} & \ldots & g_{2, j_{m}} \\
\vdots & \vdots & \ddots & \vdots \\
g_{k, j_{1}} & g_{k, j_{2}} & \ldots & g_{k, j_{m}}
\end{array}\right) .
$$

The recovery of $\mathbf{u}$ reduces to solving the system of $m=k+\delta$ linear equations in $k$ unknowns

$$
\tilde{\mathbf{G}}^{T} \mathbf{u}^{T}=\mathbf{y}^{T} .
$$

The solution of (4) can be obtained (e.g., via Gaussian elimination) if and only if $\operatorname{rank}(\tilde{\mathbf{G}})=k$.

Assuming $\mathcal{C}^{\prime}$ being MDS, the system is solvable with probability 1 if, among the $m$ received symbols, at least $k$ have indexes in $\{1,2, \ldots, n\}$, i.e., if at least $m^{\prime} \geq k$ symbols produced by the linear block encoder have been received. Let us consider the less trivial case where $m^{\prime}<k$ among the $m$ received symbols have indexes in $\{1,2, \ldots, n\}$. We can partition $\tilde{\mathbf{G}}^{T}$ as

$$
\tilde{\mathbf{G}}^{T}=\left(\begin{array}{c}
\tilde{\mathbf{G}}^{\prime T} \\
\tilde{\mathbf{G}}^{\prime \prime T}
\end{array}\right)=\left(\begin{array}{cccc}
g_{1, j_{1}} & g_{2, j_{1}} & \ldots & g_{k, j_{1}} \\
g_{1, j_{2}} & g_{2, j_{2}} & \ldots & g_{k, j_{2}} \\
\vdots & \vdots & \ddots & \vdots \\
g_{1, j_{m^{\prime}}} & g_{2, j_{m^{\prime}}} & \ldots & g_{k, j_{m^{\prime}}} \\
\hline g_{1, j_{m^{\prime}+1}} & g_{2, j_{m^{\prime}+1}} & \ldots & g_{k, j_{m^{\prime}+1}} \\
g_{1, j_{m^{\prime}+2}} & g_{2, j_{m^{\prime}+2}} & \ldots & g_{k, j_{m^{\prime}+2}} \\
\vdots & \vdots & \ddots & \vdots \\
g_{1, j_{m}} & g_{2, j_{m}} & \ldots & g_{k, j_{m}}
\end{array}\right) .
$$

The MDS property of $\mathcal{C}^{\prime}$ assures that $\operatorname{rank}\left(\tilde{\mathbf{G}}^{\prime}\right)=m^{\prime}$, i.e., the first $m^{\prime}$ rows of $\tilde{\mathbf{G}}^{T}$ are linearly independent. Note that the $m^{\prime \prime} \times k$ matrix $\tilde{\mathbf{G}}^{\prime \prime T}$ (with $m^{\prime \prime}=m-m^{\prime}$ ) can be modeled as a random matrix whose elements are uniformly distributed in $\mathbb{F}_{q}$. It follows that the matrix in (5) can be put (via column permutations over $\tilde{\mathbf{G}}^{T}$ and row permutations/combinations over $\tilde{\mathbf{G}}^{\prime T}$ ) in the form

$$
\hat{\mathbf{G}}^{T}=\left(\begin{array}{l|l}
\mathbf{I} & \mathbf{A} \\
\hline \mathbf{0} & \mathbf{B}
\end{array}\right),
$$

where $\mathbf{I}$ is the $m^{\prime} \times m^{\prime}$ identity matrix, $\mathbf{0}$ is a $m^{\prime \prime} \times m^{\prime}$ all-0 matrix, and $\mathbf{A}, \mathbf{B}$ have respective sizes $m^{\prime} \times\left(k-m^{\prime}\right)$ and $m^{\prime \prime} \times\left(k-m^{\prime}\right)$. Note that the lower part of $\hat{\mathbf{G}}^{T}$ given by $(\mathbf{0} \mid \mathbf{B})$ is obtained by adding to each row of $\tilde{\mathbf{G}}^{\prime \prime T}$ a linear combination of rows from $\tilde{\mathbf{G}}^{\prime T}$, in a way that the $m^{\prime}$ leftmost columns of $\tilde{\mathbf{G}}^{\prime \prime T}$ are zeroed-out. It follows that the statistical properties of $\tilde{\mathbf{G}}^{\prime \prime T}$ are inherited by the $m^{\prime \prime} \times\left(k-m^{\prime}\right)$ submatrix $\mathbf{B}$, whose elements are hence uniformly distributed in $\mathbb{F}_{q}$. It follows that (4) is solvable if and only if $\mathbf{B}$ is full rank, i.e., if and only if $\operatorname{rank}(\mathbf{B})=k-m^{\prime}$.

\section{A. An Efficient Decoding Algorithm}

We assume next the case where the MDS code is a $(n, k)$ generalized Reed-Solomon (GRS) code with transposed generator matrix in Vandermonde form

$$
\mathbf{G}^{\prime T}=\left(\begin{array}{cccc}
1 & \beta_{1} & \cdots & \beta_{1}^{k-1} \\
1 & \beta_{2} & \cdots & \beta_{2}^{k-1} \\
\vdots & \vdots & \ddots & \vdots \\
1 & \beta_{n} & \cdots & \beta_{n}^{k-1}
\end{array}\right),
$$

where $\beta_{i}, i=1, \ldots, n$, are $n$ distinct non-zero elements of $\mathbb{F}_{q}$. Efficient decoding can be achieved by taking advantage of the structure of $\mathbf{G}^{\prime} .^{3}$ In fact, a Vandermonde matrix can be inverted with quadratic complexity [20]-[24]. This property has been widely exploited for efficient decoding of GRS over erasure channels [25]-[28]. In the following, we first review an efficient method for the inversion of a Vandermonde matrix based on the LU factorization [21]. Then, we apply the algorithm of [21] to the decoding of the proposed concatenated scheme.

1) Vandermonde Matrices and Their Inverse: Let us consider a $\gamma \times \gamma$ Vandermonde matrix

$$
\mathbf{V}=\left(\begin{array}{cccc}
1 & x_{1} & \cdots & x_{1}^{\gamma-1} \\
1 & x_{2} & \cdots & x_{2}^{\gamma-1} \\
\vdots & \vdots & \ddots & \vdots \\
1 & x_{\gamma} & \cdots & x_{\gamma}^{\gamma-1}
\end{array}\right)
$$

where $x_{i}, i=1, \ldots, \gamma$, are $\gamma$ distinct non-zero elements of $\mathbb{F}_{q}$. In the following, $\gamma$ will be referred to as the degree of the Vandermonde matrix.

The inverse of a $\mathbf{V}$ matrix can be efficiently computed according to [21] by means of two recursions. In particular, the inverse matrix $\mathbf{V}^{-1}$ can be obtained as

$$
\mathbf{V}^{-1}=\mathbf{U}^{-1} \mathbf{L}^{-1}
$$

where $\mathbf{U}$ is an upper triangular matrix whereas $\mathbf{L}$ is a lower triangular matrix. The coefficients $l_{i, j}$ of $\mathbf{L}^{-1}$ are given by

$$
l_{i, j}=\prod_{h=1, h \neq j}^{i} \frac{1}{x_{j}-x_{h}} \quad j \leq i, i>1
$$

\footnotetext{
${ }^{3}$ In this work we consider MDS codes based on Vandermonde matrices, but similar arguments hold for MDS codes based on Cauchy matrices.
} 
with $l_{1,1}=1$ and $l_{i, j}=0$ for $j>i$. Note that, for the $j$-th column of $\mathbf{L}^{-1}$, the elements below the main diagonal can be computed according to the recursion

$$
l_{i, j}=\frac{l_{i-1, j}}{x_{j}-x_{i}}
$$

for $i=j+1, \ldots, \gamma$, after computing $l_{j, j}$. Similarly, the coefficients $u_{i, j}$ of $\mathbf{U}^{-1}$ are given by

$$
u_{i, j}= \begin{cases}u_{i-1, j-1}-u_{i, j-1} x_{j-1} & j>i>1 \\ -u_{i, j-1} x_{j-1} & j>i, i=1\end{cases}
$$

with $u_{i, i}=1$ and $u_{i, j}=0$ for $j<i$. The complexity of computing $\mathbf{L}^{-1}$ and $\mathbf{U}^{-1}$ is $\mathcal{O}\left(\gamma^{2}\right)$.

Let us denote with $\mathscr{J}^{\prime}=\left\{j_{1}, j_{2}, \ldots, j_{m^{\prime}}\right\}$ any set of $m^{\prime} \leq$ $n$ indexes of rows of $\mathbf{G}^{\prime T}$. Consider the square submatrix $\mathbf{V}$ of $\mathbf{G}^{\prime T}$ composed by the $m^{\prime}$ rows (shortened to their first $m^{\prime}$ elements) of $\mathbf{G}^{\prime T}$ with indexes in $\mathscr{J}^{\prime}$,

$$
\mathbf{V}=\left(\begin{array}{cccc}
1 & \beta_{j_{1}} & \cdots & \beta_{j_{1}-1}^{m^{\prime}-1} \\
1 & \beta_{j_{2}} & \cdots & \beta_{j_{2}-1}^{m^{\prime}-1} \\
\vdots & \vdots & \ddots & \vdots \\
1 & \beta_{j_{m^{\prime}}} & \cdots & \beta_{j_{m^{\prime}}}^{m^{\prime}-1}
\end{array}\right)
$$

Note that $\mathbf{V}$ is always a Vandermonde matrix of degree $m^{\prime}$, with elements $x_{i}^{t-1}=\beta_{j_{i}}^{t-1}$, for $i, t=1, \ldots, m^{\prime}$. This observation leads to the following decoding algorithm.

2) Decoding Algorithm: Decoding can be performed with complexity $\mathcal{O}\left(k^{2}\right)$ (equivalently, with a $\mathcal{O}(k)$ cost) if $m^{\prime} \geq k$ symbols from the MDS code have been received. In fact, this is the complexity of inverting a Vandermonde matrix of degree $k$. If $m^{\prime}=0$, the decoding complexity is equivalent to that of LRFC decoder, thus cubic in $k$ (resulting in a $\mathcal{O}\left(k^{2}\right)$ cost), which is the complexity of applying the GE algorithm to solve a linear system of at least $k$ equations in $k$ unknowns.

Let us consider the case where $0<m^{\prime}<k$ symbols of the MDS code have been collected, among the $m \geq k$ received symbols. We can define $m^{\prime}$ as a fraction of $k, m^{\prime}=\xi k$, with $0<\xi<1$. The matrix $\tilde{\mathbf{G}}^{T}$ can be written as

$$
\tilde{\mathbf{G}}^{T}=\left(\begin{array}{l|l}
\mathbf{V} & \mathbf{A} \\
\hline \mathbf{B} & \mathbf{C}
\end{array}\right)
$$

where $\mathbf{V}$ is a Vandermonde matrix of degree $m^{\prime}$, whereas $\mathbf{A}$, $\mathbf{B}, \mathbf{C}$ have respective sizes $m^{\prime} \times\left(k-m^{\prime}\right),\left(m-m^{\prime}\right) \times m^{\prime}$, $\left(m-m^{\prime}\right) \times\left(k-m^{\prime}\right)$. An efficient decoding algorithm can be derived by inverting $\mathbf{V}$ according to the algorithm presented in Section III-A1. Given the matrix $\mathbf{V}^{-1}, \tilde{\mathbf{G}}^{T}$ can be multiplied by a full-rank matrix $\mathbf{M}$, with

$$
\mathbf{M}=\left(\begin{array}{c|c}
\mathbf{V}^{-1} & \mathbf{0} \\
\hline \mathbf{0} & \mathbf{I}
\end{array}\right),
$$

I being a $\left(m-m^{\prime}\right) \times\left(m-m^{\prime}\right)$ identity matrix, leading to the matrix depicted in Fig. 2. Accordingly, (4) is modified as

$$
\mathbf{M} \cdot \tilde{\mathbf{G}}^{T} \cdot \mathbf{u}^{T}=\mathbf{M} \cdot \mathbf{y}^{T} .
$$

The complexity of multiplying the $m^{\prime} \times m^{\prime}$ matrix $\mathbf{V}^{-1}$ with the matrix $\mathbf{A}$, leading to the $m^{\prime} \times\left(k-m^{\prime}\right)$ matrix $\mathbf{A}^{\prime}$, is $\mathcal{O}\left(m^{\prime 2}\left(k-m^{\prime}\right)\right)$, which is the complexity of performing standard matrix multiplications.
Referring to Fig. 2, the $i$-th row of the matrix $\mathbf{B}$ (for $\left.i=1, \ldots, m-m^{\prime}\right)$ can be zeroed-out by adding to it a linear combination of the $m^{\prime}$ rows of $\left(\mathbf{I} \mid \mathbf{A}^{\prime}\right)$. The complexity of zeroing-out $\mathbf{B}$ is $\mathcal{O}\left(\left(m-m^{\prime}\right) m^{\prime}\left(k-m^{\prime}\right)\right)$, and the resulting system matrix is depicted in Fig. 3. In fact, $\mathbf{B}$ is a random matrix with entries uniformly distributed in $\mathbb{F}_{q}$. Due to the linear combinations performed to zero-out the matrix $\mathbf{B}$, the matrix $\mathbf{C}$ results in in a new matrix $\mathbf{C}^{\prime}$. Thus, a GE step is performed on the matrix $\mathbf{C}^{\prime}$ in order to recover the $k-m^{\prime}$ symbols involved in the lower part of the system of equations with complexity $\mathcal{O}\left(\left(k-m^{\prime}\right)^{3}\right)$. Finally, backsubstitution is applied in order to recover the $m^{\prime}$ symbols involved in the upper part of the system of equations with complexity $\mathcal{O}\left(m^{\prime}\left(k-m^{\prime}\right)\right)$.

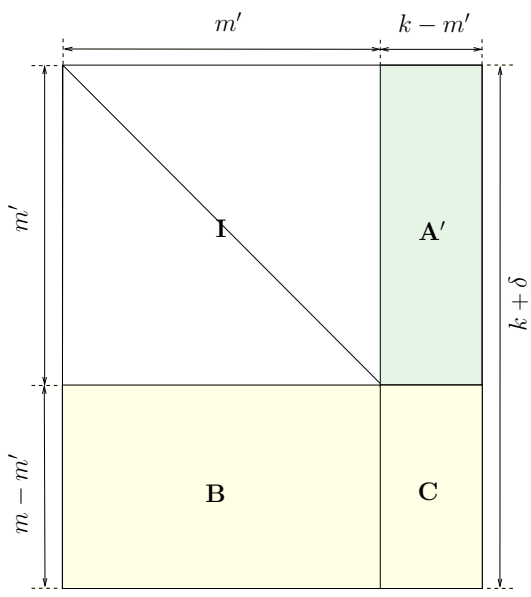

Fig. 2. Matrix of the system of equations in (5) after the multiplication with M.

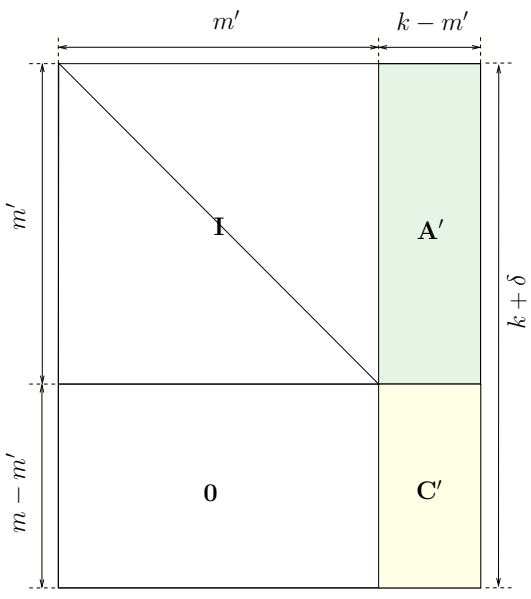

Fig. 3. Matrix of the system of equations in (5) with $\mathbf{B}=\mathbf{0}$.

Since $m^{\prime}$ is a fraction of $k$, the complexity of the proposed algorithm is $\mathcal{O}\left(k^{3}\right)$ (i.e., $\mathcal{O}\left(k^{2}\right)$ cost). However, the constant hidden by the $\mathcal{O}$-notation becomes smaller as $m^{\prime}$ approaches $k$ (in the limit case where $m^{\prime}=k$, the decoding complexity is actually quadratic in $k$ ). 


\section{Performance Analysis}

Based on the bounds (1), tight upper and lower bounds for the decoding failure probability of the fountain coding scheme can be derived in case of a memory-less erasure channel. The decoding failure probability $P_{f}=\operatorname{Pr}\{F\}$, where $F$ denotes the decoding failure event is defined as the probability that the source block $\mathbf{u}$ cannot be recovered out of a set of received symbols. We focus on the case where the linear block code used in concatenation with the LRFC is maximum distance separable (MDS). When binary codes will be used, we assume $(k+1, k)$ single parity-check (SPC) codes. When operating on higher order finite fields, we consider GRS codes.

Suppose now that an encoded sequence $\mathbf{c}$ composed of $l \geq$ $n$ symbols is transmitted over an erasure channel with erasure probability of $\epsilon{ }^{4}$ The probability that at least $k$ symbols out of the $n$ symbols produced by the linear block code encoder are received is given by

$$
Q(\epsilon)=\sum_{i=k}^{n}\left(\begin{array}{c}
n \\
i
\end{array}\right)(1-\epsilon)^{i} \epsilon^{n-i} .
$$

Hence, with a probability $P(\epsilon)=1-Q(\epsilon)$ the receiver would need to collect symbols encoded by the LRFC encoder to recover the source block. Assuming that the receiver collects $m=k+\delta$ symbols, out of which only $m^{\prime}<k$ have been produced by the linear block encoder, the conditional decoding failure probability can be expressed as

$$
\operatorname{Pr}\left\{F \mid m^{\prime}, m^{\prime}<k, \delta\right\}=\operatorname{Pr}\left\{\operatorname{rank}(\mathbf{B})<k-m^{\prime}\right\} .
$$

Note that $\mathbf{B}$ is a $m^{\prime \prime} \times\left(k-m^{\prime}\right)=\left(k+\delta-m^{\prime}\right) \times\left(k-m^{\prime}\right)$ random matrix having $\delta$ rows in excess w.r.t. the number of columns. We can thus replace (8) in (1), obtaining the bounds

$$
q^{-\delta-1} \leq \operatorname{Pr}\left\{F \mid m^{\prime}, m^{\prime}<k, \delta\right\}<\frac{1}{q-1} q^{-\delta} .
$$

Observing that the the bounds in (1) are independent from the size of the matrix (i.e., they depend only on the overhead), the conditioning on $m^{\prime}$ can be removed from (9), leaving

$$
q^{-\delta-1} \leq \operatorname{Pr}\left\{F \mid m^{\prime}<k, \delta\right\}<\frac{1}{q-1} q^{-\delta} .
$$

The failure probability can be written as a function of $\delta$ and $\epsilon$ as

$$
\begin{aligned}
P_{f}(\delta, \epsilon)= & \operatorname{Pr}\left\{F \mid m^{\prime}<k, \delta\right\} \operatorname{Pr}\left\{m^{\prime}<k\right\} \\
& +\operatorname{Pr}\left\{F \mid m^{\prime} \geq k, \delta\right\} \operatorname{Pr}\left\{m^{\prime} \geq k\right\}
\end{aligned}
$$

where $\operatorname{Pr}\left\{F \mid m^{\prime} \geq k, \delta\right\}=0$ (since at least $k$ symbols output by the MDS code encoder have been collected) and $\operatorname{Pr}\left\{m^{\prime}<\right.$ $k\}=P(\epsilon)$. It results that

$$
P(\epsilon) q^{-\delta-1} \leq P_{f}(\delta, \epsilon)<P(\epsilon) \frac{1}{q-1} q^{-\delta} .
$$

From an inspection of (1) and (11), one can note how the bounds on the failure probability of the concatenated scheme are scaled down by a factor $P(\epsilon)$, which is a monotonically increasing function of $\epsilon$. It follows that, when the channel conditions are bad (i.e., large $\epsilon$ ) $P(\epsilon) \rightarrow 1$, and the bounds

\footnotetext{
${ }^{4}$ The case $l<n$ is not considered since it is equivalent to shortening the linear block code.
}

in (11) tend to coincide with the bounds in (1). When the channel conditions are good (i.e., small $\epsilon$ ), most of the time $m^{\prime} \geq k$ symbols produced by the linear block encoder are received, leading to a decoding success (recall the assumption of MDS code). In these conditions, $P(\epsilon) \ll 1$, and according to the bounds in (11) the failure probability may decrease by several orders of magnitude. Since the probability of decoding failure of the concatenated scheme is a function of the erasure probability, the scheme is not universal anymore. More specifically, at low channel erasure probabilities the proposed scheme will outperform universal (random) LRFCs, whereas for large erasure probabilities it will perform as a universal LRFC. Fig. 4 shows the probability of decoding failure as a function of the number of overhead symbols for a concatenated code built using a $(11,10)$ SPC code over $\mathbb{F}_{2}$. It can be observed how, for lower erasure probabilities, the gain in performance of the concatenated code with respect to a LRFC increases. For $\epsilon=0.01$ the decoding failure probability is more than 2 orders of magnitude lower than that of a LRFC. Fig. 5 shows the probability of decoding failure vs. the number of overhead symbols for the concatenation of a $(15,10) \mathrm{RS}$ and a LRFC over $\mathbb{F}_{16}$. The performance of the concatenated code is compared with that of the LRFC built on the same field for different erasure probabilities. In this case the decrease in terms of probability of decoding failure is even more evident than the one of the binary case. For a channel with an erasure probability $\epsilon=0.05$, the probability of decoding failure of the concatenated scheme is 4 orders of magnitude lower than that of the LRFC.

The analysis provided in this section is also valid if the LRFC is replaced by a Raptor code. ${ }^{5}$ In order to calculate the performance of such a concatenated code one has to replace in (10) the term $\operatorname{Pr}\left\{F \mid m^{\prime}<k, \delta\right\}$ by the probability of decoding failure of the Raptor code. Also in this case, the failure probability of the concatenated scheme is reduced by a factor $P(\epsilon)$ with respect to that of the Raptor code.

\section{Numerical Results}

Fig. 6 shows the probability of decoding failure $P_{f}$, as a function of the overhead $\delta$, obtained via Monte Carlo simulations. The results refer to a concatenation of a $(15,10) \mathrm{RS}$ code with a LRFC over $\mathbb{F}_{16}$, for a channel erasure probability $\epsilon=0.1$. The results are compared with the bounds of (11). As expected, the simulation results tightly match the bounds. Fig. 7 shows the simulation results for a concatenated code using a $(11,10)$ parity check code over $\mathbb{F}_{2}$, and a channel with an erasure probability $\epsilon=0.1$. Also in this case, the results are remarkably close to the bounds.

The performance of the concatenated scheme in a system with a large receivers population has been performed. The number of receivers is denoted by $N$. We considered the erasure channels from the transmitter to the different receivers to be independent, albeit with an identical erasure probability

\footnotetext{
${ }^{5}$ As observed in [6], short Raptor codes over $\mathbb{F}_{q}$ show performance close to those of LRFCs constructed over the same field, down to moderate-low error rates. We therefore expect that the results attained by the proposed concatenation could be closely approached by replacing the non-binary LRFC with a non-binary Raptor code.
} 


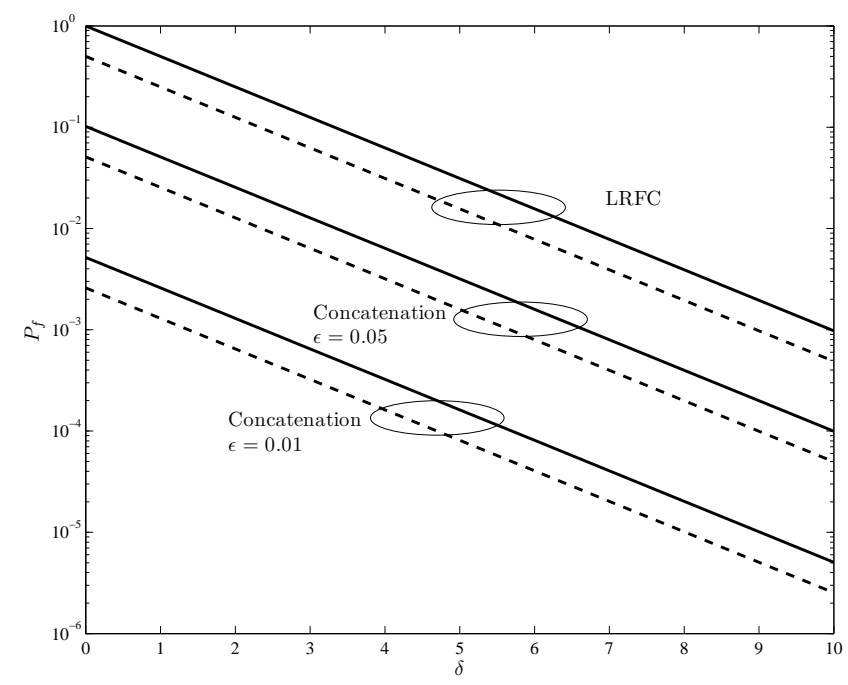

Fig. 4. $P_{f}(\delta, \epsilon)$ vs. overhead for a concatenated code built using a $(11,10)$ SPC code over $\mathbb{F}_{2}$ for different values of $\epsilon$. Upper bounds are represented by solid lines and lower bounds are represented by dashed lines.

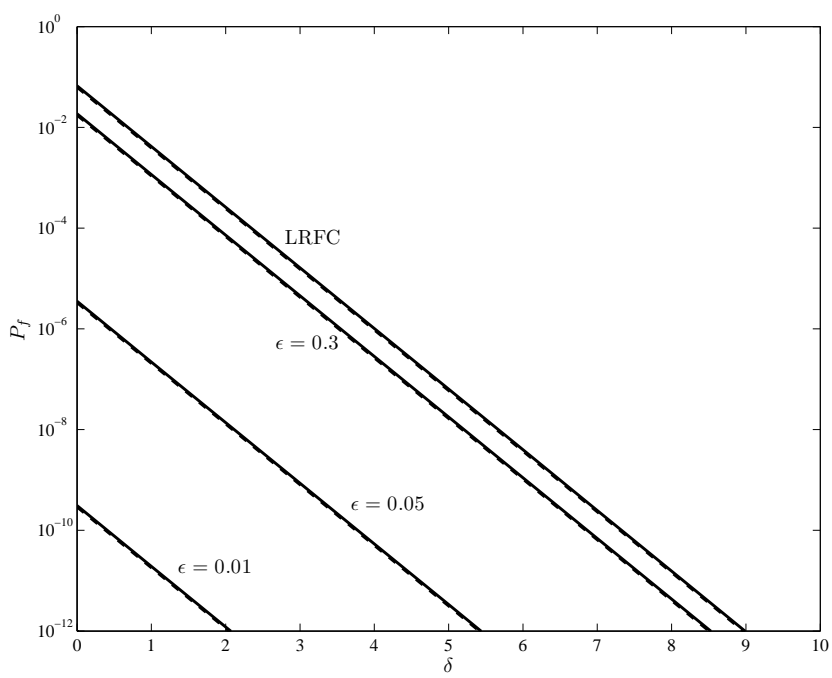

Fig. 5. $P_{f}(\delta, \epsilon)$ vs. overhead for a concatenated code built using a $(15,10)$ RS over $\mathbb{F}_{16}$ for different values of $\epsilon$. Upper bounds are represented by solid lines and lower bounds are represented by dashed lines.

$\epsilon$. Furthermore, we assumed that the receivers send an acknowledgement to the transmitter whenever they successfully decode the source block. Ideal (error- and delay-free) feedback channels have been considered. After retrieving all the acknowledgments, the transmitter stops encoding additional symbols from the source block. We denote next by $\Delta$ the number of symbols transmitted by the sender, in excess with respect to $k$. We refer to $\Delta$ as the transmission overhead. When $k+\Delta$ symbols have been transmitted, the probability that a specific receiver gathers exactly $m$ symbols is

$$
S(\Delta, m)=\left(\begin{array}{c}
k+\Delta \\
m
\end{array}\right)(1-\epsilon)^{m} \epsilon^{k+\Delta-m} .
$$

The probability of decoding failure at the receiver given that

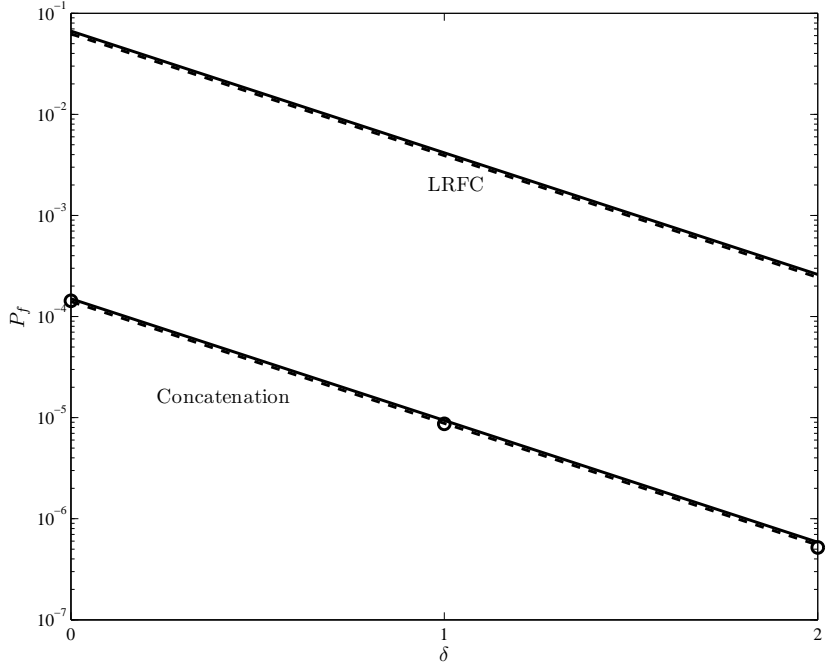

Fig. 6. $P_{f}(\delta, \epsilon)$ vs. overhead for a the concatenation of a $(15,10)$ RS and LRFC over $\mathbb{F}_{16}$ and $\epsilon=0.1$. Upper and lower bounds are represented by solid and dashed lines, respectively. The markers 'o' denote simulations.

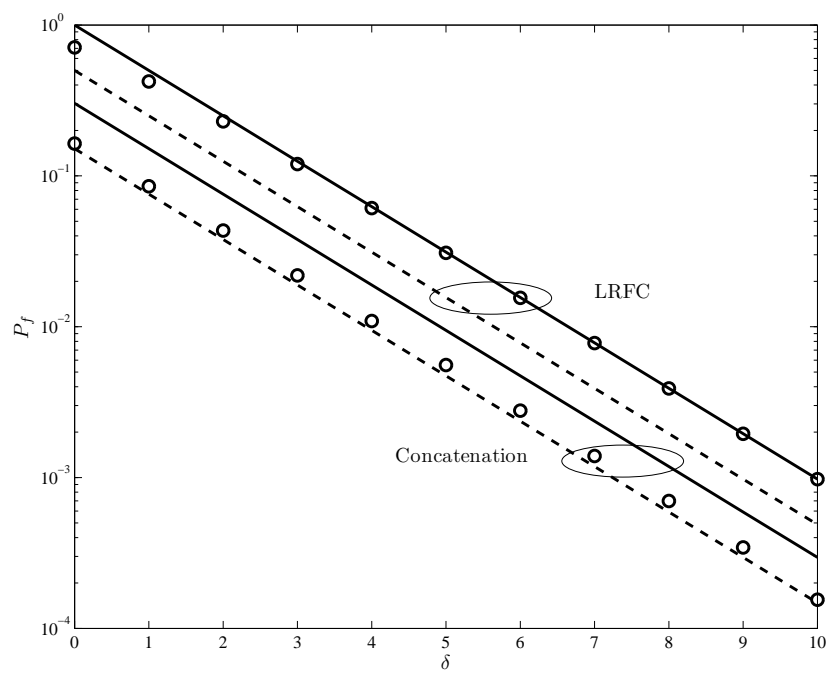

Fig. 7. $P_{f}(\delta, \epsilon)$ vs. overhead symbols for a the concatenation of a $(11,10)$ SPC code and a LRFC over $\mathbb{F}_{2}$ and $\epsilon=0.1$. Upper bounds are represented by solid lines and lower bounds are represented by dashed lines. The points marked with 'o' denote actual simulations.

the transmitter has sent $k+\Delta$ symbols is hence

$$
\begin{aligned}
P_{e}= & \sum_{m=0}^{k-1} S(\Delta, m)+ \\
& +\sum_{m=k}^{k+\Delta} S(\Delta, m) P_{f}(\delta=m-k, \epsilon) .
\end{aligned}
$$

The probability that at least one receiver is not able to decode the source block is thus

$$
P_{E}(N, \Delta, \epsilon)=1-\left(1-P_{e}\right)^{N}
$$

Observe that $P_{E}(N, \Delta, \epsilon)$ can be easily bounded by means of (11). Following this approach, we compare the performance of the proposed concatenation to that of LRFCs and to that of idealized fountain codes. We assume a system with $N=10^{4}$ 


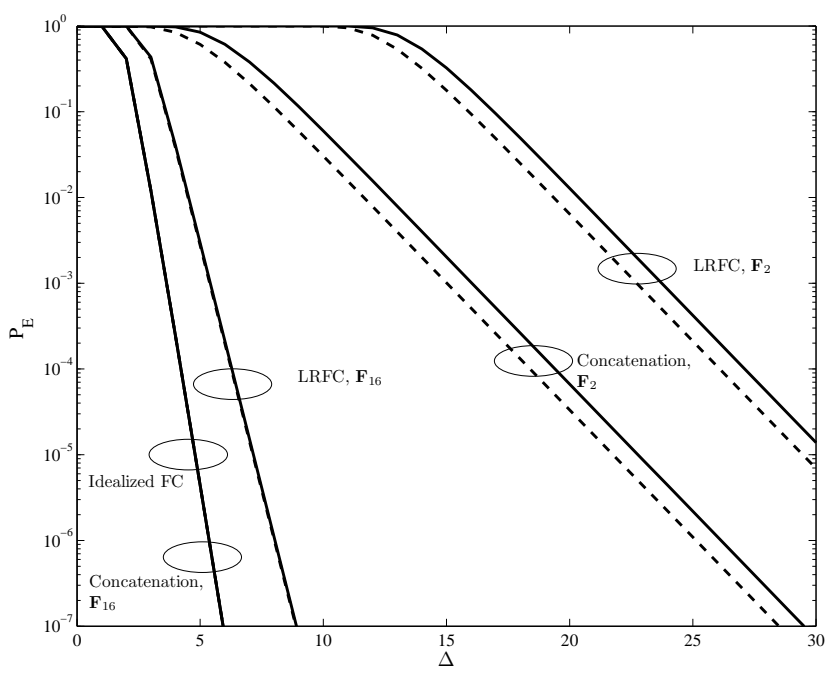

Fig. 8. $\quad P_{E}$ vs. overhead at the transmitter in a system with $N=10000$ receivers and $\epsilon=0.01$. Results are shown for different fountain codes: LRFC in $\mathbb{F}_{2}$, LRFC in $\mathbb{F}_{16}$, concatenation of a $(11,10)$ SPC code with a LRFC code in $\mathbb{F}_{2}$, and a concatenation of a $(15,10)$ RS code and a LRFC code over $\mathbb{F}_{16}$.

receivers and a channel with an erasure probability $\epsilon=0.01$. The performance of LRFC codes over $\mathbb{F}_{2}$ and $\mathbb{F}_{16}$ is depicted in Fig. 8 together with that of two concatenated schemes: A concatenation of a $(11,10)$ SPC code with a LRFC code over $\mathbb{F}_{2}$, and a concatenation of a $(15,10)$ RS code and a LRFC code over $\mathbb{F}_{16}$. It can be seen how the concatenated scheme in $\mathbb{F}_{2}$ outperforms the binary LRFC. To achieve $P_{E}=10^{-4}$ the concatenated scheme needs only $\Delta=20$ overhead symbols whereas the LRFC requires a transmission overhead $\Delta=27$. In the case of a field order 16 , the concatenated code shows a performance very close to that of an idealized fountain code.

\section{Vi. Conclusions}

A novel coding scheme has been introduced. The scheme consists of a parallel concatenation of a MDS block code with a LRFC code, both constructed over the same field. The performance of the concatenated coding scheme has been analyzed through derivation of tight bounds on the probability of decoding failure as a function of the receiver overhead. It has been shown how under ML decoding the concatenated scheme performs as well as LRFC codes in channels characterized by high erasure probabilities, whereas it provides failure probabilities lower than those of LRFC codes by several orders of magnitude at moderate/low erasure probabilities. An efficient decoding algorithm has been introduced for the case in which the generator matrix of the MDS block code is in Vandermonde form. Finally, the complexity of the proposed decoding algorithm has been analyzed, showing remarkable complexity savings at moderate/low erasure probability regimes.

\section{APPENDIX}

\section{Performance in the Finite Rate Setting}

Fountain codes are often used in a finite rate setting as conventional erasure correcting codes [13], [29]. In this context, the main advantage in the use of fountain codes with respect to block erasure correcting codes stems from the possibility of adapting code rate and block length to the transmission needs (e.g., channel conditions) in a flexible manner. In the following, we derive tight upper bounds on the block error probability for the codes proposed in the paper, for the general case where the block code $\mathcal{C}^{\prime}$ is not MDS.

In order to characterize the block error probability of a code under ML decoding we first seek for the weight-enumerator function (WEF) of the code. The coding scheme proposed in this work is a parallel concatenation of a linear block code and a LRFC, which for a finite rate setting is a random generator matrix code. Let us denote as $\mathscr{C}\left(\mathcal{C}^{\prime}, k, l, q\right)$ the ensemble of codes obtained by a parallel-concatenation of a $(n, k)$ linear block code over $\mathbb{F}_{q}, \mathcal{C}^{\prime}$, with all possible realizations of a LRFC, where $k$ is the number of source symbols, $l$ is the total number of output symbols and $q$ is the finite field order. The rate for the codes in the ensemble is, therefore, $r=k / l$. We denote as $\mathcal{A}_{i}(X)$ the conditional output-weight enumerator function (C-OWEF) averaged over the ensemble $\mathscr{C}\left(\mathcal{C}^{\prime}, k, l, q\right)$ conditioned to the input source block having weight $i$,

$$
\mathcal{A}_{i}(X)=\sum_{w=1}^{l} \mathcal{A}_{i, w} X^{w}
$$

where $\mathcal{A}_{i, w}$ is the average number of codewords of Hamming weight $w$ produced by Hamming weight- $i$ inputs. For the ensemble of parallel-concatenated codes the average C-OWEF can be written as

$$
\mathcal{A}_{i}(X)=\frac{A_{i}^{\mathcal{C}^{\prime}}(X) \mathcal{A}_{i}^{\mathscr{L}(k, h, q)}(X)}{\left(\begin{array}{l}
k \\
i
\end{array}\right)},
$$

where $A_{i}^{\mathcal{C}^{\prime}}(X)$ is the C-OWEF of the linear block code, and $\mathcal{A}_{i}^{\mathscr{L}(k, h, q)}(X)$ is the average C-OWEF of the ensemble $\mathscr{L}(k, h, q)$, being $\mathscr{L}(k, h, q)$ the ensemble of linear block codes over $\mathbb{F}_{q}$ with $k \times h$ generator matrix $\mathbf{G}^{\prime \prime}$, with $h=l-n$. Assuming $A_{i}^{\mathcal{C}^{\prime}}(X)$ known $^{6}$, the derivation of $\mathcal{A}_{i, w}$ reduces to the calculation of $\mathcal{A}_{\dot{L}}^{\mathscr{L}(k, h, q)}(X)$.

We denote by $\mathcal{A}_{i, w}^{\mathscr{L}(k, h, q)}$ the average number of codewords of Hamming weight $w$ produced by Hamming weight $i$ inputs for the ensemble $\mathscr{L}(k, h, q)$ which is given by:

$$
\mathcal{A}_{i, w}^{\mathscr{L}(k, h, q)}=\left(\begin{array}{c}
k \\
i
\end{array}\right)\left(\begin{array}{c}
h \\
w
\end{array}\right) p_{i}^{w}\left(1-p_{i}\right)^{h-w},
$$

where $p_{i}(q)$ the probability for each of the $h$ output symbols having a non-zero value conditioned to having an input of Hamming weight $i$. Assuming the coefficients of $\mathbf{G}^{\prime \prime}$ are picked with uniform probability over $\mathbb{F}_{q}$, we have that ${ }^{7}$

$$
\begin{aligned}
& p_{i}=\frac{q-1}{q}, i \neq 0 \\
& p_{i}=0 \quad, i=0 .
\end{aligned}
$$

Finally, from the average C-OWEF, $\mathcal{A}_{i}(X)$, the average WEF $\mathcal{A}(X)$ can be computed as

$$
\mathcal{A}(X)=\sum_{w} \mathcal{A}_{w} X^{w}
$$

\footnotetext{
${ }^{6}$ In general, the derivation of the C-OWEF $A_{i}^{\mathcal{C}^{\prime}}(X)$ for a code is not trivial, unless the code $\mathcal{C}^{\prime}$ (or its dual code) has small dimension [30].

${ }^{7}$ Note that when $i=0$ the encoder input is given by the all-zero word. Thus, the encoder output is zero with probability 1 due to the linearity of the code ensemble $\mathscr{L}(k, h, q)$.
} 
being $A_{w}$ the average number of codewords of Hamming weight $w, \mathcal{A}_{w}=\sum_{i} \mathcal{A}_{i, w}$.

The average WEF of the concatenated ensemble can be used now to derive tight upper bounds on the expected block error probability for the codes of the ensemble. Let $\mathcal{C}$ be a linear block code belonging to the ensemble $\mathscr{C}\left(\mathcal{C}^{\prime}, k, l, q\right)$. The block error probability averaged over the ensemble can be upper bounded as [31], [32]

$$
\begin{aligned}
& \mathbb{E}_{\mathscr{C}\left(\mathcal{C}^{\prime}, k, l, q\right)}\left[P_{B}(\mathcal{C}, \epsilon)\right] \leq P_{B}^{(\mathrm{S})}(l, k, \epsilon) \\
& +\sum_{e=1}^{l-k}\left(\begin{array}{l}
l \\
e
\end{array}\right) \epsilon^{e}(1-\epsilon)^{l-e} \min \left\{1, \sum_{w=1}^{e}\left(\begin{array}{c}
e \\
w
\end{array}\right) \frac{\mathcal{A}_{w}}{\left(\begin{array}{c}
l \\
w
\end{array}\right)}\right\}
\end{aligned}
$$

where $P_{B}^{(\mathrm{S})}(l, k, \epsilon)$ is the Singleton bound

$$
P_{B}^{(\mathrm{S})}(l, k, \epsilon)=\sum_{e=l-k+1}^{l}\left(\begin{array}{l}
l \\
e
\end{array}\right) \epsilon^{e}(1-\epsilon)^{l-e} .
$$

As an example, consider the concatenation where the block code is a binary $(63,57)$ Hamming code. Recall that the C-OWEF $\mathcal{A}_{i}(X)$ of a $\left(n=2^{t}-1, k=n-t\right)$ Hamming code [33] can be derived from

$$
\begin{aligned}
\mathcal{A}(x, X)= & \frac{(1+x)^{2^{t-1}-t-1}}{2^{t}} \times\left(2^{t}(1-x)^{2^{t-1}-t}(1-x X)^{t}\right. \\
& \left.-(1-x)^{2^{t-1}}(1+X)^{t}+(1+x)^{2^{t-1}}(1+X)^{t}\right)
\end{aligned}
$$

where $\mathcal{A}(x, X)=\sum_{i} \mathcal{A}_{i}(X) x^{i}$. Fig. 9 shows the average distance spectrum of the concatenated code. The markers represent the distance spectrum of the concatenated code, whereas the solid lines represent the average distance spectrum for the ensemble of LRFC with rate equal to the concatenated scheme. Fig. 10 shows the upper bounds on the expected block error probability of the ensemble, $P_{B}$, as a function of the channel erasure probability $\epsilon$ for different coding rates. The solid lines represent the upper bound on the block error probability in (14), and the dashed black and dotted red lines represent respectively the Berlekamp random coding bound [11]

$$
\begin{aligned}
P_{B}^{(\mathrm{B})}(l, k, \epsilon) & =\sum_{e=l-k+1}^{l}\left(\begin{array}{l}
l \\
e
\end{array}\right) \epsilon^{e}(1-\epsilon)^{l-e} \\
& +\sum_{e=1}^{l-k}\left(\begin{array}{l}
l \\
e
\end{array}\right) \epsilon^{e}(1-\epsilon)^{l-e} 2^{-(l-k-e)}
\end{aligned}
$$

which is an upper bound on the average block error probability of random codes, and the Singleton bound, which provides the block error probability of MDS codes. The markers represent the results of Monte Carlo simulations. In order to obtain average results for the ensemble, the block error probability was averaged over 1000 different LRFC realizations. The bound in (14) is very tight, as expected. Results for three different rates are shown in the figure. The highest rate corresponds to the use of the Hamming code alone, and the other two rates are $r=0.8$ and $r=0.5$. While for the Hamming code the performance lies in between the one of random codes and the one of MDS codes, as the code rate decreases the performance of the scheme gets closer to the
Berlekamp random coding bound, which means that for low rates our scheme performs almost as a random code.

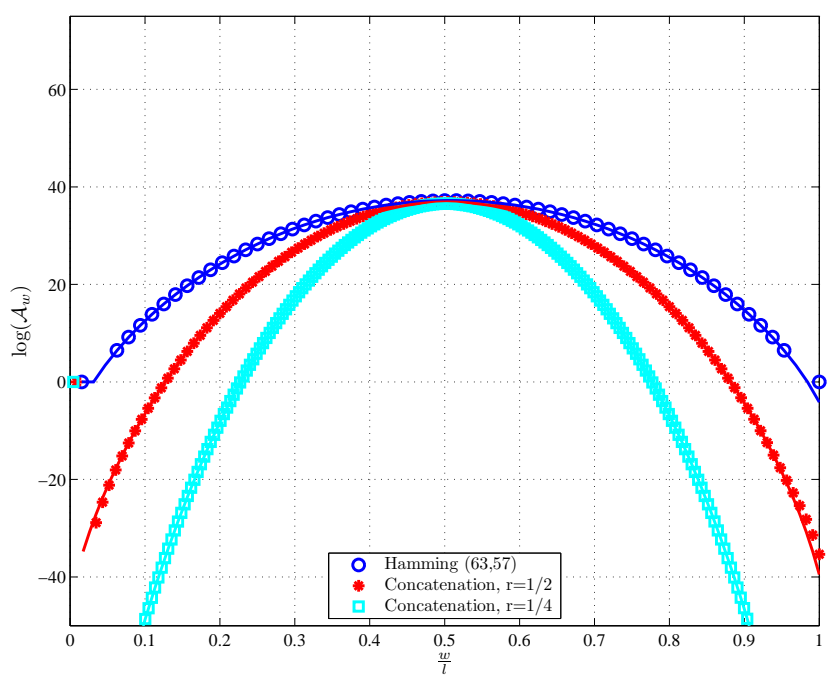

Fig. 9. $\log \left(\mathcal{A}_{w}\right)$ vs. $\frac{w}{l}$ for the concatenation of a $(63,57)$ Hamming code with a LRFC code in $\mathbb{F}_{2}$. The round markers represent the distance spectrum for the Hamming code. The asterisks and squares represent the distance spectrum of the concatenated scheme with rates $r=\frac{1}{2}$ and $r=\frac{1}{4}$ respectively. The solid lines represent the average distance spectrum for a random generator matrix code (equivalent to a LRFC in a finite rate setting).

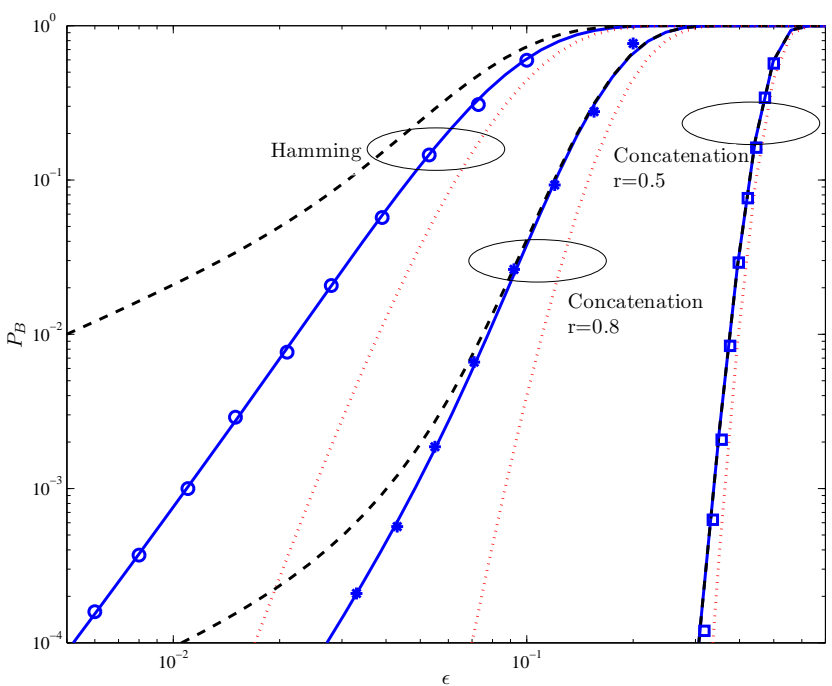

Fig. 10. $P_{B}$ vs. erasure probability $\epsilon$ for the concatenation of a $(63,57)$ Hamming code with a LRFC code in $\mathbb{F}_{2}$. The markers represent the result of Monte Carlo simulations. The solid line represents the upper bound in [31], and the black dashed and red dotted lines represent the Berlekamp random coding bound and the Singleton bound respectively.

\section{REFERENCES}

[1] J.Metzner, "An improved broadcast retransmission protocol," IEEE Trans. Commun., vol. 32, pp. 679-683, Jun. 1984.

[2] J. Byers, M. Luby, and M. Mitzenmacher, "A digital fountain approach to reliable distribution of bulk data," IEEE J. Select. Areas Commun., vol. 20, no. 8, pp. 1528-1540, Oct. 2002.

[3] M. Luby, "LT codes," in Proc. of the 2002 Annual IEEE Symp. on Foundations of Computer Science, pp. 271-282. 
[4] M. Shokrollahi, "Raptor codes," IEEE Trans. Inform. Theory, vol. 52, no. 6, pp. 2551-2567, Jun. 2006.

[5] J. K. Sundararajan, D. Shah, and M. Medard, "ARQ for network coding," in Proc. of the 2009 IEEE Int. Symp. on Inf. Theory, pp. 1651-1655.

[6] G. Liva, E. Paolini, and M. Chiani, "Performance versus overhead for fountain codes over $\mathbb{F}_{q}$," IEEE Commun. Lett., vol. 14, no. 2, pp. 178180, Feb. 2010

[7] G. Liva, C. Kissling, and C. Hausl, "A simple coded ARQ for satellite broadcasting," Journal of Communication and Networks, vol. 12, no. 6 , pp. 577-581, Dec. 2010.

[8] F. Lázaro Blasco and G. Liva, "On the concatenation of non-binary random linear fountain codes with maximum distance separable codes," in Proc. of the 2011 IEEE Int. Conf. on Commun., pp. 1 -5.

[9] B. Schotsch, H. Schepker, and P. Vary, "The performance of short random linear fountain codes under maximum likelihood decoding," in Proc. of the 2011 IEEE Int. Conf. on Commun., pp. 1-5.

[10] B. Schotsch, R. Lupoaie, and P. Vary, "The performance of low-density random linear fountain codes over higher order galois fields under maximum likelihood decoding," in Proc. of the 2011 Annu. Allerton Conf. on Commun., Control, and Computing, pp. $1004-1011$.

[11] E. Berlekamp, "The technology of error-correcting codes," Proc. IEEE, vol. 68, pp. 564-593, May 1980.

[12] M. Luby, A. Shokrollahi, M. Watson, T. Stockhammer, and L. Minder, "RaptorQ forward error correction scheme for object delivery," IETF, Tech. Rep., 2011, iETF Internet Draft.

[13] 3GPP TS 26.346 V6.1.0, "Technical specification group services and system aspects; multimedia broadcast/multicast service; protocols and codecs," Jun. 2005.

[14] B. A. LaMacchia and A. M. Odlyzko, "Solving large sparse linear systems over finite fields," Lecture Notes in Computer Science, vol. 537, pp. 109-133, 1991.

[15] T. Richardson and R. Urbanke, "Efficient encoding of low-density paritycheck codes," IEEE Trans. Inform. Theory, vol. 47, pp. 638-656, Feb. 2001.

[16] D. Burshtein and G. Miller, "An efficient maximum likelihood decoding of LDPC codes over the binary erasure channel," IEEE Trans. Inform. Theory, vol. 50, no. 11, pp. 2837-2844, Nov. 2004.

[17] M. Shokrollahi, S. Lassen, and R. Karp, "Systems and processes for decoding chain reaction codes through inactivation," Feb. 2005, US Patent 6,856,263.

[18] E. Paolini, G. Liva, B. Matuz, and M. Chiani, "Maximum likelihood erasure decoding of LDPC codes: Pivoting algorithms and code design," IEEE Trans. Commun., vol. 60, no. 11, pp. 3209 -3220, Nov. 2012.

[19] K. Kasai, D. Declercq, and K. Sakaniwa, "Fountain Coding via Multiplicatively Repeated Non-Binary LDPC Codes," IEEE Trans. Commun., vol. 60, no. 8, pp. 2077-2083, Aug. 2012.

[20] F. Parker, "Inverses of Vandermonde matrices," Amer. Math. Monthly, vol. 71, pp. 410-411, Apr. 1964.

[21] L. R. Turner, "Inverse of Vandermonde matrix with applications," NASA, Tech. Rep. D-3547, Aug. 1966.

[22] I.Kaufman, "The inversion of the Vandermonde matrix and the trasformation to the Jordan canonical form," IEEE Trans. Automat. Contr, vol. 14, pp. 774-777, Dec. 1969.

[23] H. Wertz, "On the numerical inversion of a recurrent problem: the Vandermonde matrix," IEEE Trans. Automat. Contr., vol. 4, no. 10, p. 492, Oct. 1965

[24] I. Gohberg and V. Olshevsky, "Fast algorithms with pre-processing for matrix-vector multiplication problems," Journal of Complexity, vol. 10, pp. 411-427, Dec. 1994.

[25] G. Forney, Concatenated codes. Cambridge, MA, USA: M.I.T. Press, 1966.

[26] R. McEliece, The theory of information and coding. Cambridge, UK: Cambridge Univ. Press, 2002.

[27] J. Brauchle and R. Koetter, "A systematic Reed-Solomon encoder with arbitrary parity positions," in Proc. of the 2009 IEEE Global Telecommun. Conf., pp. $1-4$.

[28] J. Brauchle, "On efficient recovery of erased symbols in generalized Reed-Solomon codes," in Proc. of the 2011 IEEE Int. Conf. on Commun., pp. $1-5$.

[29] ETSI TR 102993 V1.1.1, "Digital Video Broadcasting (DVB); upper layer FEC for DVB systems," Feb. 2011.

[30] F. Mac Williams and N. Sloane, The theory of error-correcting codes. North Holland Mathematical Libray, 1977.

[31] C. Di, D. Proietti, I. Telatar, T. Richardson, and R. Urbanke, "Finitelength analysis of low-density parity-check codes on the binary erasure channel," IEEE Trans. Inform. Theory, vol. 48, no. 6, pp. $1570-1579$, Jun. 2002.
[32] G. Liva, E. Paolini, and M. Chiani, "Bounds on the Error Probability of Block Codes over the q-ary Erasure Channel," IEEE Trans. Commun., vol. 61, no. 2, pp. $2201-2211$, Jun. 2013.

[33] F. Chiaraluce and R. Garello, "On the asymptotic performance of Hamming product codes," in Proc. of the 2001 International Symp. on Commun. Theory and Applications, pp. 329-334.

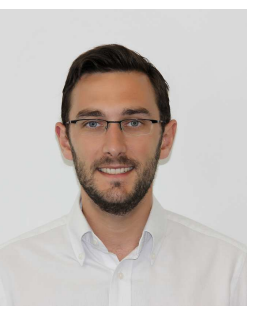

Francisco Lázaro Blasco was born in Zaragoza, Spain, in 1983. He received his Telecommunication Engineering degree from the Universidad de Zaragoza, Spain, in 2006. In 2008 he joined the Institute for Communications and Navigation at the German Aerospace Center (DLR), as a research scientist, where he is working toward his $\mathrm{PhD}$. His main research interests are rate-less codes and multiuser communications with focus on satellite and space communications. During the past years he has been involved in many national and international projects. He is an IEEE student member and servers as IEEE reviewer for Journals and Conferences.

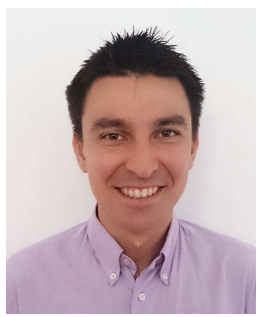

Giuliano Garrammone was born in Pesaro, Italy, in 1983. He received the Master of Science degree in Electrical Engineering from the University of Bologna, Italy, in 2008. While working toward the Master degree, he was an international student at the Polytechnic University of Valencia, in Spain and at the University of California Santa Barbara, in the US. He works for the Institute of Communications and Navigation of the German Aerospace Center (DLR) in Germany, since 2008, as a scientific researcher. His main research interests include channel coding and network coding for wireless communications systems, in particular for satellite and space communications, with focus on low-density paritycheck codes, rate-less codes, and Reed-Solomon codes. During the past years he has been involved in several national and international projects. Among the others, he has been working on the Germany-funded Network Coding Project (NEXT), where he has contributed to the design, implementation and validation of a transmission system based on non-binary fountain codes.

$\mathrm{He}$ is an IEEE student member and he serves IEEE as a reviewer for Transactions, Journals and Conferences, as well as a technical program committee member.

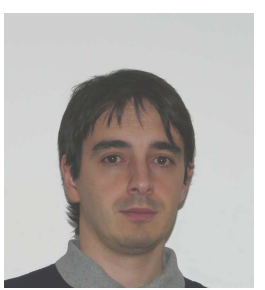

Gianluigi Liva (M'08) was born in Spilimbergo, Italy, in 1977. He received the M.S. and the Ph.D. degrees in electrical engineering from the University of Bologna (Italy) in 2002 and 2006, respectively.

His main research interests include satellite communication systems, random access techniques and error control coding. Since 2003 he has been investigating channel codes for high data rate space missions, in collaboration with the European Space Agency (ESA). In 2004 and 2005 he was at the University of Arizona, investigating the design of lowcomplexity coding systems for space communications. He is currently with the Institute of Communications and Navigation of the German Aerospace Center (DLR). He is / he has been active in the CCSDS, DVB-S2 and in the DVB-RCS standardizations. In 2010 he has been lecturing for channel coding at the Institute for Communications Engineering (LNT) of the Technische Universität München (TUM). In 2012 and 2013 he has been lecturing for channel coding at the Nanjing University of Science and Technology (China).

Dr. Liva is IEEE member and he serves IEEE as reviewer for Transactions, Journals and Conferences. Since 2013 he is Editor for IEEE COMMUNICATIONS LETTERS. 